Vittalle - Revista de Ciências da Saúde v. 32, n. 2 (2020) 173-179

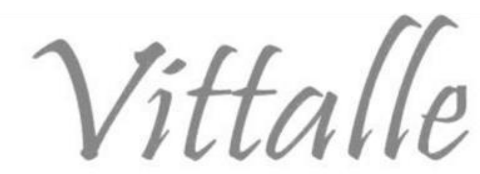

\title{
Vasculite do sistema nervoso central secundária a Neurocriptococose - Relato de Caso
}

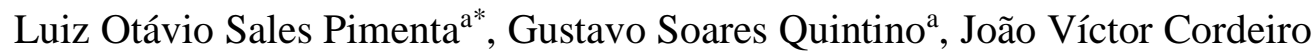 \\ Rodrigues $^{\mathrm{b}}$, Euldes Mendes Junior ${ }^{\mathrm{c}}$, Evandro Barbosa dos Anjos ${ }^{\mathrm{a}}$ \\ aFaculdades Unidas do Norte de Minas (FUNORTE), Montes Claros, MG, Brasil \\ ${ }^{b}$ Universidade Federal de Ouro Preto (UFOP), Ouro Preto, MG, Brasil \\ ${ }^{\mathrm{c}}$ Centro de Esclerose Múltipla de Montes Claros, Montes Claros, MG, Brasil
}

\section{Histórico do Artigo Recebido em: 28/12/2019 \\ Aceito em: $25 / 08 / 2020$}

Palavras-chave: Meningoencefalite; criptococose; meningite criptocócica; vasculite; infarto cerebral

Keywords:

Meningoencephalitis; cryptococcosis; meningitis cryptococcal; vasculitis; cerebral infarction.

\begin{abstract}
RESUMO
A meningoencefalite criptocócica é causada por fungos do gênero Cryptococcus, podendo infectar indivíduos imunossuprimidos e imunocompetentes. A infecção do sistema nervoso central (SNC) é a forma mais comum da criptococose extrapulmonar, podendo resultar em alta morbimortalidade. A doença criptocócica pode se manifestar como abscessos, meningite, granulomas e infartos e as condições que causam imunossupresão aumentam o risco de infecção do SNC. O diagnóstico é baseado em punção lombar e culturas, devendo-se ter a infecção por Cryptococcus como diagnóstico diferencial do acidente vascular cerebral, principalmente em indivíduos imunocomprometidos. O objetivo desse estudo é relatar o caso de um paciente com vasculite do SNC secundária a uma meningoencefalite criptocócica. Trata-se de uma paciente do sexo feminino, 34 anos, que fazia uso de medicação imunossupressora para controle da Doença de Crohn. Desenvolveu uma vasculite do SNC na vigência de infecção por Cryptococcus neoformans, manifestando-se com infarto cerebral, cujos achados foram confirmados nos exames de ressonância magnética do encéfalo e angiorressonância cerebral. A punção lombar apresentou o padrão típico da neurocriptococose. Tinta da China e cultura confirmaram o diagnóstico etiológico. Porém, mesmo com a terapêutica adequada, a paciente evoluiu para o óbito. Sabe-se que esse quadro de vasculite do SNC associada à infecção criptocócica é raro. Portanto, as manifestações inespecíficas dessa condição e seu início subagudo ou crônico geralmente podem levar a atrasos no diagnóstico e tratamento adequados. Assim, é importante o conhecimento dessa apresentação na neurocriptococose, uma vez que o diagnóstico e conduta precoces propiciam redução na morbimortalidade do paciente.
\end{abstract}

\section{Central nervous system vasculitis secondary to Neurocriptococcosis - Case Report}

\section{ABSTRACT}

Cryptococcal meningoencephalitis is caused by fungi of the genus Cryptococcus, and can infect immunosuppressed and immunocompetent individuals. Central nervous system (CNS) infection is the most common form of extrapulmonary cryptococcosis, which can result in high morbidity and mortality. Cryptococcal disease can manifest itself as abscesses, meningitis, granulomas and infarcts and the conditions that cause immunosuppression increase the risk of infection of the CNS. The diagnosis is based on lumbar puncture and cultures, and Cryptococcus infection should be considered as a differential diagnosis of stroke, especially in immunocompromised individuals. The objective of this study is to report the case of a patient with CNS vasculitis secondary to cryptococcal meningoencephalitis. This is a 34-year-old female patient who used immunosuppressive medication to control Crohn's disease. He developed CNS vasculitis in the presence of Cryptococcus neoformans infection, manifesting himself with cerebral infarction, the findings of which were confirmed in the magnetic resonance imaging of the brain and cerebral angiography. The lumbar puncture showed the typical pattern of neurocriptococcosis. Chinese ink and culture confirmed the etiological diagnosis. However, even with the proper therapy, the patient died. It is known that this CNS vasculitis associated with cryptococcal infection is rare. Therefore, nonspecific manifestations of this condition and its subacute or chronic onset can often lead to delays in proper diagnosis and treatment. Thus, it is important to know this presentation in neurocriptococcosis, since the early diagnosis and conduct provide a reduction in the patient's morbidity and mortality.

\footnotetext{
* Autor correspondente: luizotaviocdz@hotmail.com (Pimenta L.O.S.)
} 


\section{Introdução}

A meningoencefalite criptocócica é uma infecção do sistema nervoso central (SNC) causada pelo fungo levedura encapsulada do gênero Cryptococcus, que pode infectar indivíduos imunocompetentes e imunossuprimidos (1). Condições imunossupressoras que aumentam o risco de infecção do SNC incluem: infecção pelo vírus da imunodeficiência humana (HIV), leucemia, imunossupressores, síndrome da falência orgânica e problemas imunes inatos, como a imunodeficiência variável comum (2).

$\mathrm{O}$ acometimento pelo fungo é mais comum em sítios pulmonares, sendo a infecção do SNC a forma mais comum da doença criptocócica extrapulmonar, podendo resultar em alta morbimortalidade (1). Pode se manifestar no sistema nervoso como abscessos, meningite, granulomas e infartos (causados por vasculite de pequenos vasos) (2). Algumas infecções do SNC como: herpes-zóster, tuberculose e citomegalovírus são mais conhecidas como causas de vasculite e infarto cerebral, em comparação à criptococose, que apesar de alta afinidade pelo SNC raramente apresenta esta manifestação $(3,4)$.

Sintomas como início súbito de um déficit neurológico focal que dura mais que 24 horas, associado com tomografia computadorizada (TC) de crânio evidenciando lesões em áreas correspondentes aos déficits apresentados (3) sugerem infarto cerebral. Na vasculite por Cryptococcus, infartos ocorrem principalmente em região de cápsula interna, tálamo e núcleos da base, manifestando-se com: distúrbios visuais e de linguagem, diminuição do nível de consciência, paresias, ataxia, cefaleia, vertigem e outros sintomas neurológicos $(1,3)$.

O diagnóstico da neurocriptococose é feito pela punção lombar e cultura, sendo dificultado pelo fato de não ser rotineiro o estudo do líquor em pacientes com acidente vascular cerebral (AVC) (1,2). A análise do líquor em pacientes com meningoencefalite criptocócica revela altas pressões de abertura, elevação dos níveis de proteínas e de contagem de células (com predomínio de linfócitos), com baixos valores de glicose (2). A pesquisa do fungo é feita a partir da coloração com tinta nanquim e culturas do líquor $(1,4)$. Exames radiológicos como TC e ressonância magnética (RM) contribuem para visualização das áreas de infarto, ressaltando-se o valor da angiografia com evidências de vasculite, contribuindo no diagnóstico sindrômico $(1,2,3)$.

Deve-se ter a infecção por Cryptococcus como diagnóstico diferencial nos casos suspeitos de AVC, principalmente em indivíduos imunocomprometidos (2). O não reconhecimento precoce pode levar a um atraso diagnóstico, tratamento inadequado e piora do prognóstico $(1,2)$.

Dessa forma, o objetivo deste estudo é relatar o caso de uma paciente com quadro de vasculite do SNC secundária a neurocriptococose, apresentando os sintomas da paciente, o método diagnóstico, a propedêutica e a terapêutica empregadas. Este relato se faz de extrema importância, por se tratar de uma apresentação pouco comum da neurocriptococose, em que o atraso diagnóstico acarreta grande morbimortalidade aos pacientes.

O projeto deste trabalho foi avaliado e aprovado pelo Comitê de Ética e Pesquisa com parecer de aprovação de número: 3.606.537.

\section{Relato do caso}

Mulher, 34 anos de idade, natural de Montes Claros, Minas Gerais, procura serviço hospitalar com queixa de tetraparesia de início súbito. Concomitantemente ao quadro, a paciente também queixava de dificuldade para articular frases e apresentava desvio do 
olhar conjugado para a esquerda. Refere uma história de cefaleia holocraniana de moderada intensidade iniciada há cerca de 5 dias do início dos sintomas atuais. Nega etilismo, tabagismo e uso de drogas ilícitas. Nega quadros infecciosos prévios aos sintomas atuais. Como comorbidade, ela relatava ser portadora de doença de Crohn, fazendo uso de Azatioprina e Infliximabe.

O exame inicial da paciente na sala de emergência revelou uma frequência cardíaca de 110 batimentos por minuto, pressão arterial de 130/90 mmHg e temperatura axilar de $37.3^{\circ} \mathrm{C}$. A paciente estava alerta, bem orientada e cooperativa (apresentava 15 pontos na escala de coma de Glasgow). Sua fluência verbal era parcialmente prejudicada devido a um quadro de afasia motora. O diâmetro de suas pupilas era $3 \mathrm{~mm}$ bilateralmente, ambas responsivas ao estímulo luminoso. O exame dos nervos cranianos revelou desvio do olhar conjugado para a esquerda associado a nistagmo multidirecional. Reflexos corneopalpebral, oculocefálico e de tosse presentes. Apresentava uma tetraparesia (força muscular grau 3 globalmente) e função sensitiva preservada e simétrica. Leve ataxia em membros superiores e inferiores, com demais provas cerebelares sem alterações. Impossibilidade de avaliação da marcha em virtude da paresia de membros inferiores. Foi realizada TC de crânio, que evidenciou múltiplas lesões hipodensas em regiões corticais, subcorticais e nucleocapsulares, consistentes com regiões de infarto cerebral recente.

No dia seguinte, a paciente evoluiu com rebaixamento do nível de consciência e piora da tetraparesia, com surgimento de sinal de Babinski bilateralmente e rigidez de nuca. Procedeu-se o estudo com RM de encéfalo (Figura 1) para melhor elucidação diagnóstica, e a sequência de difusão revelou múltiplas lesões no córtex cerebral, região subcortical, cerebelar, região nucleocapsular e corpo caloso, com correspondência no mapa de coeficiente aparente de difusão. Uma angioressonância cerebral (Figura 2) foi indicada, para melhor estudo dos vasos intracranianos. Este exame evidenciou estenose de múltiplos vasos, compatível com um quadro de vasculite autoimune do SNC.

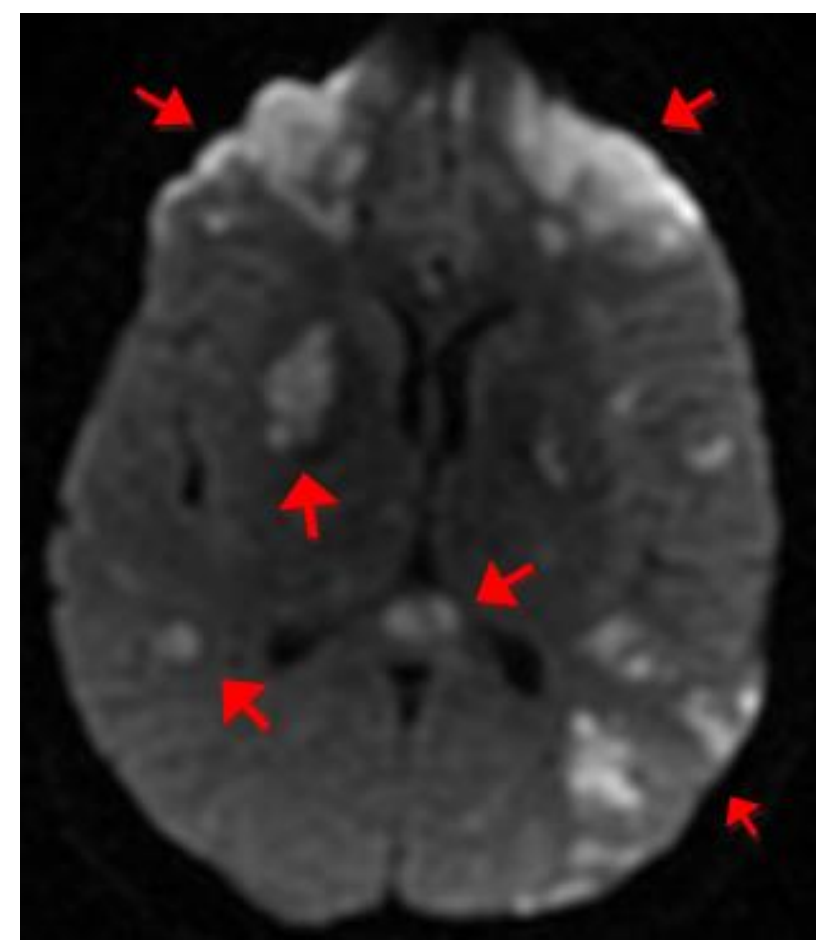

Figura 1 - Ressonância magnética de encéfalo em corte axial, sequência ponderada em difusão, evidenciando múltiplas lesões hiperintensas em córtex cerebral, região subcortical, nucleocapsular e corpo caloso. As setas estão apontando para os principais achados da imagem. 


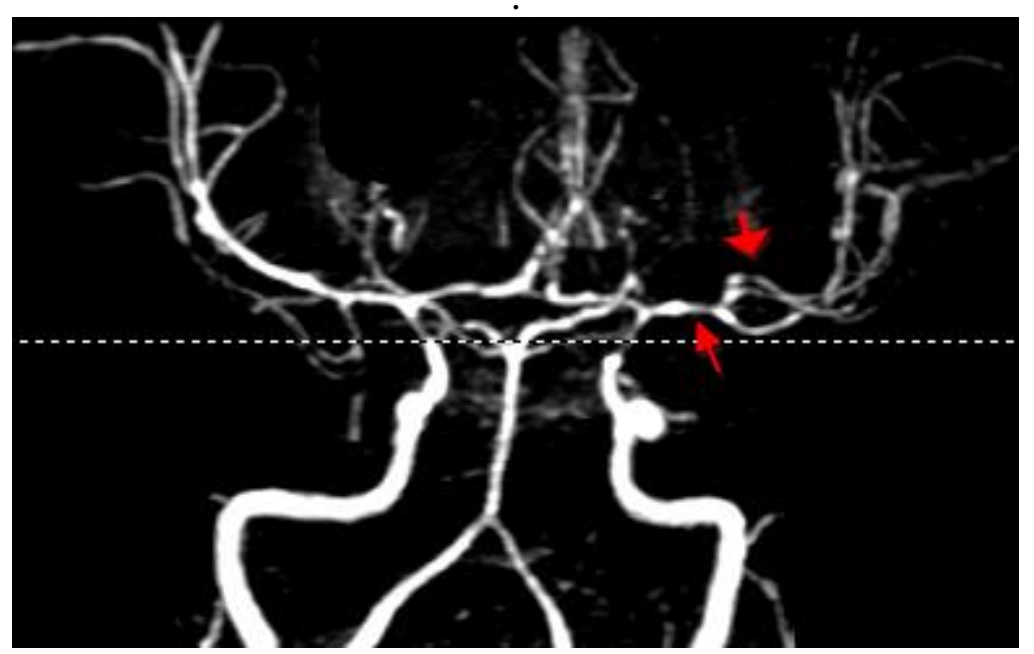

Figura 2 - Angiorressonância cerebral, fase arterial, evidenciando estenose de múltiplos vasos da circulação anterior e posterior, sugerindo um quadro de vasculite do SNC. As setas estão apontando para os principais achados da imagem.

Antes do tratamento programado com corticosteroides, uma punção lombar foi realizada para diagnósticos diferenciais. Enquanto o resultado da análise do líquor não saia, foi realizado uma pulsoterapia com Metilprednisolona, $1 \mathrm{~g} /$ dia. O líquido cefalorraquidiano (LCR) foi coletado com uma pressão de abertura de $32 \mathrm{~cm} \mathrm{H}_{2} \mathrm{O}, 68$ células (com predomínio de mononucleares), $181 \mathrm{md} / \mathrm{dL}$ de proteínas e $2 \mathrm{mg} / \mathrm{dL}$ de glicose (a glicemia capilar era $129 \mathrm{mg} / \mathrm{dL}$ ). Tendo em vista o resultado do exame que não foi compatível com a hipotése inicial de vasculite imunomediada, a pulsoterapia foi suspensa e novos exames foram solicitados.

Foi realizada pesquisa direta de fungos com tinta da China, que mostrou presença de Cryptococcus neoformans. Assim, o diagnóstico de vasculite autoimune do SNC foi alterado para vasculite infecciosa secundária à neurocriptococose. Foi iniciado então para a paciente um esquema de tratamento medicamentoso com Fluconazol e Anfotericina B Desoxicolato: durante os 14 primeiros dias estava programada a realização da terapia de indução com Fluconazol, $800 \mathrm{mg} /$ dia e Anfotericina B Desoxicolato $01 \mathrm{mg} / \mathrm{kg} / \mathrm{dia}$. Posteriormente, seguiria a terapia de manutenção apenas com o Fluconazol, $800 \mathrm{mg} / \mathrm{dia}$. Todavia, sabe-se que a Anfotericina B apresenta como efeito adverso a hipocalemia, fato que fez com que a paciente evoluísse com parada cardiorrespiratória em assistolia oito dias após o início do tratamento, quando veio a falecer.

\section{Discussão}

O relato aborda o caso de uma paciente, sexo feminino, 34 anos, que desenvolveu uma vasculite do SNC na vigência de infecção por criptococco (gênero Cryptococcus), manifestando-se com infarto cerebral. A ocorrência de infarto na vigência dessa infecção é rara, ocorrendo em $4 \%$ dos pacientes negativos para a síndrome da imunodeficiência humana (3).

A idade média de apresentação descrita em pacientes imunossuprimidos foi de 38 anos (variando de 23-60 anos), sendo a maioria em homens (2). Infecções oportunistas são frequentemente causadas por Cryptococcus, em condições que comprometem a imunidade como a infecção pelo HIV, leucemia, uso de imunossupressores e erros 
inatos aumentam o risco de desenvolver a neurocriptococose $(1,2)$. Deve se atentar para o fato de que a paciente em questão realizava uso de medicação imunossupressora (Azatioprina e Infliximabe) para controle da Doença de Crohn, atuando como fator de risco para infecções oportunistas. A neurocriptococose, em sua maior parte, acomete os pacientes imunossuprimidos, podendo, raramente, acometer indivíduos imunocompetentes, se tornando um desafio diagnóstico pela raridade dessa apresentação e suas manifestações inespecíficas (1).

A infecção do SNC é a forma mais frequente de acometimento extrapulmonar da criptococose, podendo se manifestar como granulomas, abscessos, meningite e vasculite dos vasos intracranianos $(1,2)$. Como manifestação do envolvimento do SNC da paciente em questão, a mesma desenvolveu um quadro de vasculite dos vasos intracranianos, cuja patogenia se baseia em deposição de antígeno-anticorpo, além da toxicidade direta, resultando em infarto de pequenos vasos, com acometimento de gânglios da base, tálamo e cápsula interna $(1,3)$. Esses infartos podem ainda acometer outras regiões, como substância branca profunda, córtex e regiões de vascularização dos grandes vasos cerebrais (2). Deve-se atentar para o fato de que a TC de crânio da paciente apresentava, de fato, lesões nessas áreas mais típicas de envolvimento (núcleos da base, tálamo, córtex e substância branca adjacente).

O início das manifestações pode ser subagudo ou crônico, e o infarto é mais comum nesses pacientes do que o déficit neurológico transitório (com duração menor que 24 horas e total retorno à normalidade) $(1,2)$.

É mais comum os pacientes que cursam com déficit neurológico transitório apresentarem: cefaleias, distúrbios da linguagem e da visão, em contraste com os pacientes que se manifestam com infarto, que desenvolvem, na maioria dos casos, encefalopatia (3). Destaca-se que o sintoma mais apresentado por esses pacientes foi a cefaleia, podendo ter outras manifestações inespecíficas antecedendo a dor de cabeça (1). Nos dois casos, o sinal neurológico focal mais apresentado foi hemiparesia. Além disso, outros sinais e sintomas são afasia, paralisia facial, disartria, desorientação visuoespacial e outros (3). A paciente do presente estudo apresentou cefaleia holocraniana, seguida de déficits neurológicos focais como tetraparesia e afasia motora. Evoluiu também com encefalopatia, se manifestando como rebaixamento do nível de consciência.

Embora não seja clássica a análise do líquor em pacientes com AVC, uma punção lombar deve ser realizada imediatamente diante da suspeita de uma meningoencefalite criptocócica, uma vez que o diagnóstico desta entidade se baseia em culturas e análises do LCR $(1,2)$. O estudo do líquor foi feito no caso em questão, apresentando o padrão típico encontrado nos pacientes com neurocriptococose: pressão de abertura elevada, alta celularidade (predomínio de linfócitos), glicose baixa, e proteínas elevadas (4). Tintura nankin (tinta da China) e cultura confirmam o diagnóstico etiológico para meningoecefalitecriptocócica $(1,3)$. A paciente do estudo foi submetida à pesquisa direta de fungos com tinta da China, confirmando-se o diagnóstico etiológico da infecção criptocócica.

Outros exames de imagem, como TC de crânio e angiografia cerebral, podem auxiliar o diagnóstico, evidenciando áreas de infarto cerebral e vasculite dos vasos intracranianos (1-3). A paciente estudada possuía uma TC de crânio com lesões típicas de infarto cerebral e uma angiografia demonstrando estenose dos vasos intracranianos, sugestivo de vasculite do SNC.

$\mathrm{O}$ tratamento deve se basear em combater a infecção vigente e condições ameaçadoras à vida do paciente, manejando também complicações subjacentes, como hipertensão intracraniana e hipocalemia devido ao uso de anfotericina B (1). O tratamento é 
baseado, principalmente, no uso de anfotericina B associado a Flucitosina, seguido de manutenção com fluconazol $(1,3)$. A paciente do estudo fez uso da anfotericina B desoxicolato e fluconazol, entretanto evoluiu para o óbito decorrente hipocalemia (efeito adverso do uso da anfotericina B).

Nesse cenário, observa-se que a incidência de infecções fúngicas invasivas tem aumentado, como consequência do contingente cada vez maior de pacientes com imunossupressão (5). O tratamento das infecções fúngicas com anfotericina B está associado a efeitos adversos importantes, como nefrotoxicidade. A perda de função renal é uma complicação relativamente comum, assim como outros eventos, como hipocalemia, hipomagnesemia e acidose metabólica (6). Nos músculos, a hipocalemia gera hiperpolarização do miócito tornando-o mais refratário à excitação neural e, como consequência, provoca fadiga, fraqueza muscular, e dificuldade respiratória. Nos cardiomiócitos, o mesmo distúrbio leva a um prolongamento na repolarização refletido no eletrocardiograma por depressão de ST-T e presença de ondas U e, posteriormente aumento do intervalo QT (7). Dessa forma, trata-se de um disrtúrbio hidroeletrolítico com elevado potencial de evoluir ao óbito, como o que ocorreu com a paciente do caso.

Sabe-se que a neurocriptococose que se manifesta com vasculite apresenta pior prognóstico (1), de modo que é importante considerar infecções do SNC como causas de infartos cerebrais é importante (4). O atraso diagnóstico e tratamento inadequado podem deteriorar o prognóstico do paciente, resultando em incapacidades neurológicas graves e morte (2). A paciente do nosso estudo apresentou vasculite do SNC secundária a neurocriptococose, acarretando um pior prognóstico. Houve a suspeita de infecção do SNC como causa dos infartos cerebrais assim que o estudo do líquor foi obtido. A evolução da doença da paciente em questão foi rápida, com evolução para o óbito decorrente da hipocalemia, efeito adverso da anfotericina B.

\section{Conclusão}

O relato do caso evidenciou uma paciente que apresentava aspectos clínicoradiológicos compatíveis com o diagnóstico de vasculite do SNC secundária a neurocriptococose. Apesar da adequada terapêutica empregada, a paciente evoluiu para o óbito.

Conclui-se que a vasculite do SNC associada à infecção criptocócica é rara e não está bem descrita na literatura. As manifestações inespecíficas dessa condição e seu início subagudo ou crônico geralmente levam a atrasos na identificação e tratamento adequado. Sendo assim, é de suma importância o conhecimento dessa apresentação na neurocriptococose, uma vez que o diagnóstico e conduta precoces propiciam redução na morbimortalidade do paciente. O diagnóstico é muito desafiador e geralmente depende de punção lombar e culturas, sobretudo em pacientes imunocomprometidos.

\section{Referências}

1. Oberman ZD, Patrucco L, Oderiz CC. Central Nervous System Vasculitis for Cryptococcosis in an Immunocompetent Patient. Diseases 2018; 6(3): 75.

2. Lai CH, Lin GY, Lee JT, Kao HW, Ho TH, Lin YK, et al. A Rare Case of Cryptococcus Meningoencephalitis Presenting with Acute Brainstem Infarction in an Immunocompetent Host. Neuropsychiatry (London) 2017; 07(6): 812-815.

3. Engstrom JW, Lowenstein DH, Bredesen DE. Cerebral infarctions and transiente neurologic deficits associated with acquired immunodeficiency syndrome. The American Journal of Medicine 1989; 86(5): 528-532.

4. Leite AGB, Vidal JE, Filho FB, Nogueira RS, Oliveira ACP. Cerebral infarction related to 
cryptococcal meningitis in an HIV-infected patient: case report and literature review. The Brazilian Journal of Infectious Diseases 2004; 8(2): 175-179.

5. Schlottfeldt FS, Fernandes SM, Martins DM, Cordeiro P, Fonseca CD, Watanabe M, et al. Prevenção da nefrotoxicidade da anfotericina Bpor meio do uso de fitomedicamentos. Revista da Escola de Enfermagem da USP 2015; 49(Esp): 74-79.

6. Falci DR, Pasqualotto AC. Anfotericina B: uma revisão sobre suas diferentes formulações, efeitos adversos e toxicidade. Clinical \& Biomedical Research 2015; 35 (2): 65-82.

7. Pirozzi FF, Neto DL, Cipullo JP, Pires AC. Hipocalemia persistente. Relato de caso. Revista Brasileira de Clínica Médica 2012; 10(2): 147-151. 